\title{
Estimation of Optimum Dilution in the GMAW Process Using Integrated ANN-GA
}

\author{
P. Sreeraj, ${ }^{1}$ T. Kannan, ${ }^{2}$ and Subhashis Maji ${ }^{3}$ \\ ${ }^{1}$ Department of Mechanical Engineering, Valia Koonambaikulathamma College of Engineering and Technology, \\ Trivandrum, Kerala 692574, India \\ ${ }^{2}$ SVS College of Engineering, Coimbatore, Tamilnadu 642109, India \\ ${ }^{3}$ Department of Mechanical Engineering, IGNOU, New Delhi 110068, India
}

Correspondence should be addressed to P. Sreeraj; pathiyasseril@yahoo.com

Received 28 September 2012; Revised 13 December 2012; Accepted 16 December 2012

Academic Editor: Keat Teong Lee

Copyright (c) 2013 P. Sreeraj et al. This is an open access article distributed under the Creative Commons Attribution License, which permits unrestricted use, distribution, and reproduction in any medium, provided the original work is properly cited.

To improve the corrosion resistant properties of carbon steel, usually cladding process is used. It is a process of depositing a thick layer of corrosion resistant material over carbon steel plate. Most of the engineering applications require high strength and corrosion resistant materials for long-term reliability and performance. By cladding these properties can be achieved with minimum cost. The main problem faced on cladding is the selection of optimum combinations of process parameters for achieving quality clad and hence good clad bead geometry. This paper highlights an experimental study to optimize various input process parameters (welding current, welding speed, gun angle, and contact tip to work distance and pinch) to get optimum dilution in stainless steel cladding of low carbon structural steel plates using gas metal arc welding (GMAW). Experiments were conducted based on central composite rotatable design with full replication technique, and mathematical models were developed using multiple regression method. The developed models have been checked for adequacy and significance. In this study, artificial neural network (ANN) and genetic algorithm (GA) techniques were integrated and labeled as integrated ANN-GA to estimate optimal process parameters in GMAW to get optimum dilution.

\section{Introduction}

Prevention of corrosion is a major problem in industries. Even though it cannot be eliminated completely, it can be reduced to some extent. A corrosion resistant protective layer is made over the less corrosion resistant substrate by a process called cladding. This technique not only used to improve life of engineering components but also to reduce their cost. This process is mainly now used in industries such as chemical, textiles, nuclear, steam power plants, food processing, and petrochemical industries [1].

The most accepted method employed in weld cladding is GMAW. It has got the following advantages [2]:

(i) high reliability,

(ii) all-position capability,

(iii) ease to use,

(iv) low cost, (v) high Productivity,

(vi) suitable for both ferrous and nonferrous metals,

(vii) high deposition rate,

(viii) absences of fluxes,

(ix) cleanliness and ease of mechanization.

The mechanical strength of clad metal is not only highly influenced by the composition of metal but also by clad bead shape. This is an indication of bead geometry. Figure 1 shows the clad bead geometry. It mainly depends on wire feed rate, welding speed, arc voltage, and so forth. Therefore it is necessary to study the relationship between in-process parameters and bead parameters to study clad bead geometry. Using mathematical models, it can be achieved. This paper highlights the study carried out to develop mathematical and ANN-GA models to optimize clad bead geometry, in stainless steel cladding deposited by GMAW. The experiments were 


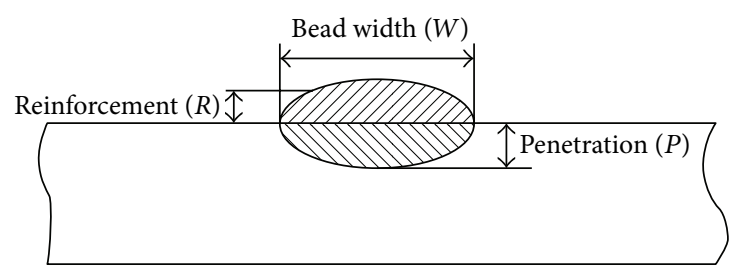

Figure 1: Clad bead geometry.

conducted based on four-factor-five-level central composite rotatable designs with full replication technique [3]. The developed models have been checked for their adequacy and significance. Again using ANN-GA, the bead parameters were optimized.

This study can be divided into three parts that are experimental analysis, analytical model, and artificial intelligence model. Experimental and analytical models can be developed using conventional and statistical models. Artificial intelligent models such as ANN and GA can be used for prediction. There are many advantages using ANN for prediction such as ANN's ability to handle nonlinear form of modeling that learn the mapping of input and output. ANN is more successful than conventional methods in terms of speed and simplicity and its capacity to learn from examples. Moreover it does not need any preliminary assumptions. Simple MATLAB Toolbox can be used for prediction.

In this study an integration system is opted for study to get an improvement in GMAW process. In this study two techniques were used: ANN is used for prediction and GA is used for optimization. These two techniques were integrated to form a new system integrated ANN-GA. It is proved that the proposed hybrid system ANN-GA can produce more significant results than conventional techniques.

\section{Experimental Procedure}

The following machines and consumables were used for the purpose of conducting experiments:

(1) a constant current gas metal arc welding machine (Invrtee V 350-PRO advanced processor with 5425 amp output range),

(2) welding manipulator,

(3) wire feeder (LF-74 Model),

(4) filler material Stainless Steel wire of $1.2 \mathrm{~mm}$ diameter (ER-308 L),

(5) gas cylinder containing a mixture of $98 \%$ argon and $2 \%$ oxygen,

(6) mild steel plate (grade IS-2062).

Test plates of size $300 \times 200 \times 20 \mathrm{~mm}$ were cut from mild steel plate of grade IS-2062, and one of the surfaces is cleaned to remove oxide and dirt before cladding. ER-308 L stainless steel wire of $1.2 \mathrm{~mm}$ diameter was used for depositing the clad beads through the feeder. Argon gas at a constant flow rate of 16 litres per minute was used for shielding. The properties of

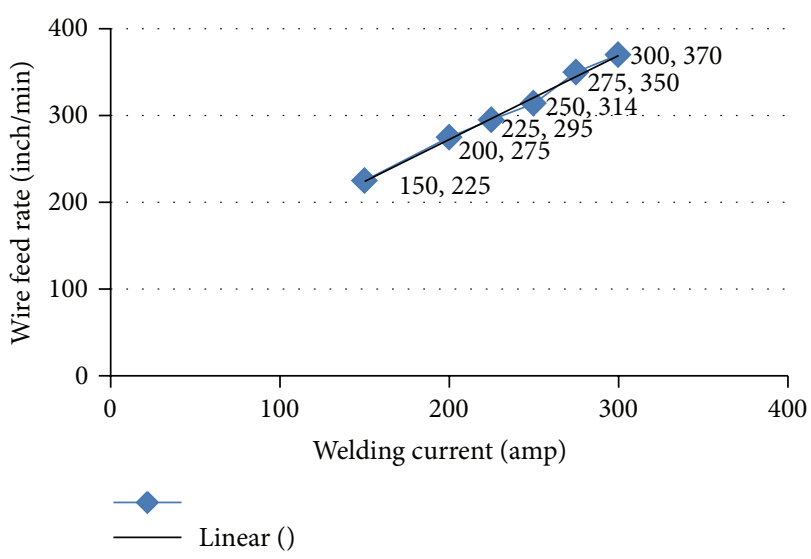

FIGURE 2: Relationship between Current and Wire Feed Rate.

base metal and filler wire are shown in Table 1. The important and most difficult parameter found from trial run is wire feed rate. The wire feed rate is proportional to current. Wire feed rate must be greater than critical wire feed rate to achieve pulsed metal transfer. The relationship found from trial run is shown in (1). The formula derived is shown in Figure 2:

wire feed rate $=0.96742857 *$ current +79.1 .

The selection of the welding electrode wire is based on the matching of the mechanical properties and physical characteristics of the base metal, weld size, and existing electrode inventory [4]. The candidate material for cladding which has excellent corrosion resistance and weldability is stainless steel. It has chloride stress corrosion cracking resistance and strength significantly greater than other materials. It has good surface appearance, good radiographic standard quality, and minimum electrode wastage. Experimental design procedure used for this study is shown in Figure 3 and important steps are briefly explained.

\section{Plan of Investigation}

The research work was planned to be carried out in the following steps [5]:

(1) identification of factors and responses,

(2) finding limits of process variables,

(3) development of design matrix,

(4) conducting experiments as per design matrix, 
TABLE 1: Chemical composition of base metal and filler wire.

\begin{tabular}{lccccccccc}
\hline \multirow{2}{*}{ Materials } & & \multicolumn{3}{c}{ Elements wt\% } & Al & Cr & Mo & Ni \\
& C & SI & Mn & P & S & - & - \\
IS 2062 & 0.150 & 0.160 & 0.870 & 0.015 & 0.016 & 0.031 & - & 19.52 & 0.75 \\
ER 308L & 0.03 & 0.57 & 1.76 & 0.021 & 0.008 & - & 10.02 \\
\hline
\end{tabular}

TABLE 2: Welding parameters and their levels.

\begin{tabular}{lcccccc}
\hline \multirow{2}{*}{ Parameters } & \multirow{2}{*}{ Unit } & Notation & \multicolumn{3}{c}{ Factor levels } \\
& & & -2 & -1 & 0 & 1 \\
\hline Welding current & A & $I$ & 200 & 225 & 250 & 275 \\
Welding speed & $\mathrm{mm} / \mathrm{min}$ & $\mathrm{S}$ & 150 & 158 & 166 & 174 \\
Contact tip to work distance & $\mathrm{mm}$ & $N$ & 10 & 14 & 18 & 22 \\
Welding gun angle & Degree & $T$ & 70 & 80 & 90 & 182 \\
Pinch & - & Ac & -10 & -5 & 0 & 100 \\
\hline
\end{tabular}

(5) recording the responses,

(6) development of mathematical models,

(7) checking the adequacy of developed models,

(8) conducting conformity tests.

3.1. Identification of Factors and Responses. The following independently controllable process parameters were found to be affecting output parameters. These are wire feed rate $(W)$, welding speed $(S)$, welding gun angle $(T)$, contact tip to work to distance $(N)$, and pinch (Ac). The responses chosen were clad bead width $(W)$, height of reinforcement $(R)$, depth of penetration $(P)$, and percentage of dilution $(D)$. The responses were chosen based on the impact of parameters on final composite model.

The basic difference between welding and cladding is the percentage of dilution. The properties of the cladding is significantly influenced by the dilution obtained. Hence control of dilution is important in cladding where a low dilution is highly desirable. When dilution is quite low, the final deposit composition will be closer to that of filler material, and hence corrosion resistant properties of cladding will be greatly improved. The chosen factors have been selected on the basis of getting minimal dilution and optimal clad bead geometry.

Few significant research works have been conducted in these areas using these process parameters, and so these parameters were used for an experimental study.

3.2. Finding the Limits of Process Variables. Working ranges of all selected factors are fixed by conducting trial runs. This was carried out by varying one of the factors while keeping the rest of them as constant values. Working range of each process parameter was decided upon by inspecting the bead for smooth appearance without any visible defects. The upper limit of a given factor was coded as -2 . The coded value of intermediate values were calculated using (2):

$$
X_{i}=\frac{2\left[2 X-\left(X_{\max }+X_{\min }\right)\right]}{\left[\left(X_{\max }-X_{\min }\right)\right]},
$$

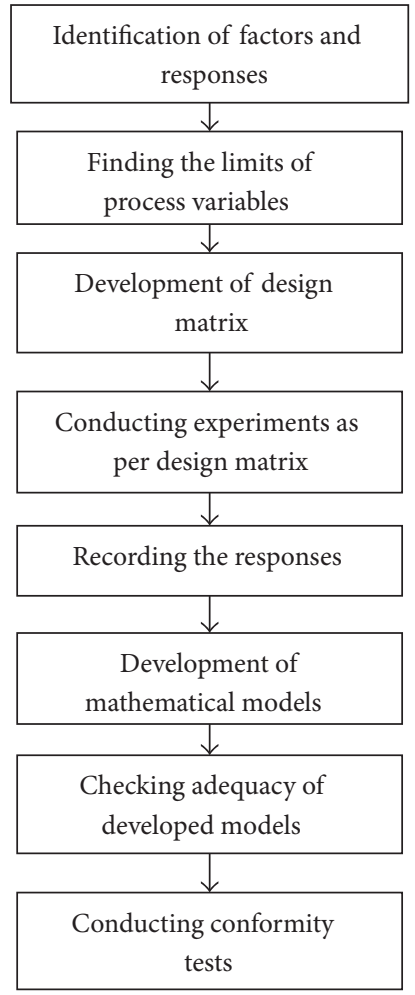

FIGURE 3: Experimental design procedure.

where $X_{i}$ is the required coded value of parameter and $X$ is any value of parameter from $X_{\min }-X_{\max }$. $X_{\min }$ is the lower limit of parameters and $X_{\max }$ is the upper limit of parameters [4].

The chosen level of the parameters with their units and notations are given in Table 2 .

3.3. Development of Design Matrix. Design matrix chosen to conduct the experiments was central composite rotatable design. The design matrix comprises full replication of $2^{5}(=32)$, factorial designs. All welding parameters in 
TABle 3: Design matrix.

\begin{tabular}{|c|c|c|c|c|c|}
\hline \multirow{2}{*}{ Trial no. } & \multicolumn{5}{|c|}{ Design matrix } \\
\hline & $I$ & $S$ & $N$ & $T$ & Ac \\
\hline 1 & -1 & -1 & -1 & -1 & 1 \\
\hline 2 & 1 & -1 & -1 & -1 & -1 \\
\hline 3 & -1 & 1 & -1 & -1 & -1 \\
\hline 4 & 1 & 1 & -1 & -1 & 1 \\
\hline 5 & -1 & -1 & 1 & -1 & -1 \\
\hline 6 & 1 & -1 & 1 & -1 & 1 \\
\hline 7 & -1 & 1 & 1 & -1 & 1 \\
\hline 8 & 1 & 1 & 1 & -1 & -1 \\
\hline 9 & -1 & -1 & -1 & 1 & -1 \\
\hline 10 & 1 & -1 & -1 & 1 & 1 \\
\hline 11 & -1 & 1 & -1 & 1 & 1 \\
\hline 12 & 1 & 1 & -1 & 1 & -1 \\
\hline 13 & -1 & -1 & 1 & 1 & 1 \\
\hline 14 & 1 & -1 & 1 & 1 & -1 \\
\hline 15 & -1 & 1 & 1 & 1 & -1 \\
\hline 16 & 1 & 1 & 1 & 1 & 1 \\
\hline 17 & -2 & 0 & 0 & 0 & 0 \\
\hline 18 & 2 & 0 & 0 & 0 & 0 \\
\hline 19 & 0 & -2 & 0 & 0 & 0 \\
\hline 20 & 0 & 2 & 0 & 0 & 0 \\
\hline 21 & 0 & 0 & -2 & 0 & 0 \\
\hline 22 & 0 & 0 & 2 & 0 & 0 \\
\hline 23 & 0 & 0 & 0 & -2 & 0 \\
\hline 24 & 0 & 0 & 0 & 2 & 0 \\
\hline 25 & 0 & 0 & 0 & 0 & -2 \\
\hline 26 & 0 & 0 & 0 & 0 & 2 \\
\hline 27 & 0 & 0 & 0 & 0 & 0 \\
\hline 28 & 0 & 0 & 0 & 0 & 0 \\
\hline 29 & 0 & 0 & 0 & 0 & 0 \\
\hline 30 & 0 & 0 & 0 & 0 & 0 \\
\hline 31 & 0 & 0 & 0 & 0 & 0 \\
\hline 32 & 0 & 0 & 0 & 0 & 0 \\
\hline
\end{tabular}

$I$ : welding current; $S$ : welding speed; $N$ : contact tip to work distance; $T$ : welding gun angle; Ac: pinch.

the intermediate levels (o) constitute the central points and combination of each welding parameters at either the highest value $(+2)$ or the lowest value $(-2)$ with other parameters of intermediate levels $(0)$ constitutes star points. 32 experimental trails were conducted to make the estimation of linear quadratic and two-way interactive effects of process parameters on clad geometry [5].

3.4. Conducting Experiments as per Design Matrix. The experiments were conducted at SVS College of Engineering, Coimbatore, India. In this work thirty-two experimental runs were allowed for the estimation of linear quadratic and twoway interactive effects correspond to each treatment combination of parameters on bead geometry as shown in Table 3 at random. At each run settings for all parameters were
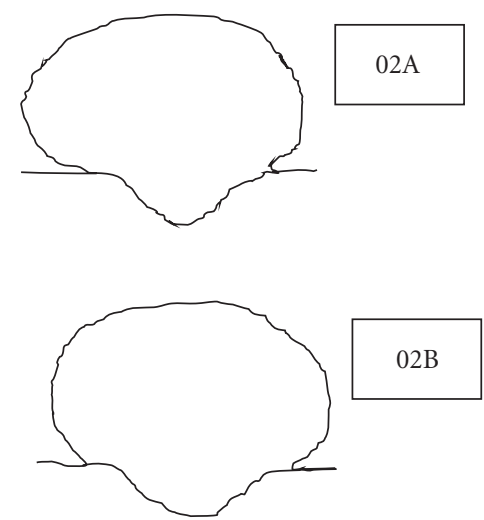

FIgURE 4: Traced Profiles (Specimen No. 2). 02A represents profile of the specimen (front side) and 02B represents profile of the specimen (rear side). The measured clad bead dimension and percentage of dilution is shown in Table 4.

disturbed and reset for next deposit. This is very essential to introduce variability caused by errors in experimental setup.

3.5. Recording of Responses. In order to measure clad bead geometry of transverse section of each weld, overlays were cut using band saw from mid length. Positions of the weld and end faces were machined and grinded. The specimen and faces were polished and etched using a $5 \%$ nital solution to display bead dimensions. The clad bead profiles were traced using a reflective type optical profile projector at a magnification of $\times 10$. Then the bead dimensions such as depth of penetration, height of reinforcement, and clad bead width were measured [6]. The traced bead profiles were scanned in order to find various clad parameters and the percentage of dilution with the help of AUTO CAD software. This is shown in Figure 4.

3.6. Development of Mathematical Models. The response function representing any of the clad bead geometry can be expressed as [7-9]

$$
Y=f(A, B, C, D, E),
$$

where $Y=$ response variable, $A=$ welding current $(I)$ in amp, $B=$ welding speed $(S)$ in $\mathrm{mm} / \mathrm{min}, C=$ contact tip to work distance $(N)$ in $\mathrm{mm}, D=$ welding gun angle $(T)$ in degrees, and $E=$ pinch (Ac).

The second-order surface response model can be expressed as below:

$$
\begin{aligned}
Y= & \beta_{0}+\beta_{1} A+\beta_{2} B+\beta_{3} C+\beta_{4} D+\beta_{5} E+\beta_{11} A^{2} \\
& +\beta_{22} B^{2}+\beta_{33} C^{2}+\beta_{44} D^{2}+\beta_{55} E^{2}+\beta_{12} A B \\
& +\beta_{13} A C+\beta_{14} A D+\beta_{15} A E+\beta_{23} B C+\beta_{24} B D \\
& +\beta_{25} B E+\beta_{34} C D+\beta_{35} C E+\beta_{45} D E,
\end{aligned}
$$

where $\beta_{0}$ is the free term of the regression equation, the coefficient $\beta_{1}, \beta_{2}, \beta_{3}, \beta_{4}$ and $\beta_{5}$ are linear terms, the coefficients, $\beta_{11}, \beta_{22}, \beta_{33}, \beta_{44}$, and $\beta_{55}$ quadratic terms, and the coefficients 
TABle 4: Design matrix and observed values of clad bead geometry.

\begin{tabular}{|c|c|c|c|c|c|c|c|c|c|}
\hline \multirow{2}{*}{ Trial no. } & \multicolumn{5}{|c|}{ Design matrix } & \multicolumn{4}{|c|}{ Bead parameters } \\
\hline & $I$ & $S$ & $N$ & $T$ & Ac & $W(\mathrm{~mm})$ & $P(\mathrm{~mm})$ & $R(\mathrm{~mm})$ & $D(\%)$ \\
\hline 1 & -1 & -1 & -1 & -1 & 1 & 6.9743 & 1.67345 & 6.0262 & 10.72091 \\
\hline 2 & 1 & -1 & -1 & -1 & -1 & 7.6549 & 1.9715 & 5.88735 & 12.16746 \\
\hline 3 & -1 & 1 & -1 & -1 & -1 & 6.3456 & 1.6986 & 5.4519 & 12.74552 \\
\hline 4 & 1 & 1 & -1 & -1 & 1 & 7.7635 & 1.739615 & 6.0684 & 10.61078 \\
\hline 5 & -1 & -1 & 1 & -1 & -1 & 7.2683 & 2.443 & 5.72055 & 16.67303 \\
\hline 6 & 1 & -1 & 1 & -1 & 1 & 9.4383 & 2.4905 & 5.9169 & 15.96692 \\
\hline 7 & -1 & 1 & 1 & -1 & -1 & 6.0823 & 2.4672 & 5.49205 & 16.5894 \\
\hline 8 & 1 & 1 & 1 & -1 & -1 & 8.4666 & 2.07365 & 5.9467 & 14.98494 \\
\hline 9 & -1 & -1 & -1 & 1 & -1 & 6.3029 & 1.5809 & 5.9059 & 10.2749 \\
\hline 10 & 1 & -1 & -1 & 1 & 1 & 7.0136 & 1.5662 & 5.9833 & 9.707297 \\
\hline 11 & -1 & 1 & -1 & 1 & 1 & 6.2956 & 1.58605 & 5.5105 & 11.11693 \\
\hline 12 & 1 & 1 & -1 & 1 & -1 & 7.741 & 1.8466 & 5.8752 & 11.4273 \\
\hline 13 & -1 & -1 & 1 & 1 & 1 & 7.3231 & 2.16475 & 5.72095 & 15.29097 \\
\hline 14 & 1 & -1 & 1 & 1 & -1 & 9.6171 & 2.69495 & 6.37445 & 18.54077 \\
\hline 15 & -1 & 1 & 1 & 1 & -1 & 6.6335 & 2.3089 & 5.554 & 17.23138 \\
\hline 16 & 1 & 1 & 1 & 1 & 1 & 10.514 & 2.7298 & 5.4645 & 20.8755 \\
\hline 17 & -2 & 0 & 0 & 0 & 0 & 6.5557 & 1.99045 & 5.80585 & 13.65762 \\
\hline 18 & 2 & 0 & 0 & 0 & 0 & 7.4772 & 2.5737 & 6.65505 & 15.74121 \\
\hline 19 & 0 & -2 & 0 & 0 & 0 & 7.5886 & 2.50455 & 6.4069 & 15.77816 \\
\hline 20 & 0 & 2 & 0 & 0 & 0 & 7.5014 & 2.1842 & 5.6782 & 16.82349 \\
\hline 21 & 0 & 0 & -2 & 0 & 0 & 6.1421 & 1.3752 & 6.0976 & 8.941799 \\
\hline 22 & 0 & 0 & 2 & 0 & 0 & 8.5647 & 3.18536 & 5.63655 & 22.94721 \\
\hline 23 & 0 & 0 & 0 & -2 & 0 & 7.9575 & 2.2018 & 5.8281 & 15.74941 \\
\hline 24 & 0 & 0 & 0 & 2 & 0 & 7.7085 & 1.85885 & 6.07515 & 13.27285 \\
\hline 25 & 0 & 0 & 0 & 0 & -2 & 7.8365 & 2.3577 & 5.74915 & 16.63287 \\
\hline 26 & 0 & 0 & 0 & 0 & 2 & 8.2082 & 2.3658 & 5.99005 & 16.38043 \\
\hline 27 & 0 & 0 & 0 & 0 & 0 & 7.9371 & 2.1362 & 6.0153 & 15.18374 \\
\hline 28 & 0 & 0 & 0 & 0 & 0 & 8.4371 & 2.17145 & 5.69895 & 14.82758 \\
\hline 29 & 0 & 0 & 0 & 0 & 0 & 9.323 & 3.1425 & 5.57595 & 22.8432 \\
\hline 30 & 0 & 0 & 0 & 0 & 0 & 9.2205 & 3.2872 & 5.61485 & 23.6334 \\
\hline 31 & 0 & 0 & 0 & 0 & 0 & 10.059 & 2.86605 & 5.62095 & 21.55264 \\
\hline 32. & 0 & 0 & 0 & 0 & 0 & 8.9953 & 2.72068 & 5.7052 & 19.60811 \\
\hline
\end{tabular}

$W$ : width; $P$ : penetration; $R$ : reinforcement; $D$ : dilution $\%$.

$\beta_{12}, \beta_{13}, \beta_{14}, \beta_{15}$, and so forth are the interaction terms. The coefficients were calculated using Quality America Six Sigma Software (DOE-PC IV). After determining the coefficients, the mathematical models were developed. The developed mathematical models are given as follows:

clad bead width $(W), \mathrm{mm}$

$$
\begin{aligned}
= & 8.923+0.701 A+0.388 B \\
& +0.587 C+0.040 D+0.088 E \\
& -0.423 A^{2}-0.291 B^{2}-0.338 C^{2} \\
& -0.219 D^{2}-0.171 E^{2}+0.205 A B \\
& +0.405 A C+0.105 A D+0.070 A E
\end{aligned}
$$

$$
\begin{aligned}
& -0.134 B C+0.225 B D+0.098 B E \\
& +0.26 C D+0.086 C E+0.012 D E
\end{aligned}
$$

depth of penetration $(P), \mathrm{mm}$

$$
\begin{aligned}
= & 2.735+0.098 A-0.032 B+0.389 C-0.032 D \\
& -0.008 E-0.124 A^{2}-0.109 B^{2}-0.125 C^{2} \\
& -0.187 D^{2}-0.104 E^{2}-0.33 A B+0.001 A C \\
& +0.075 A D+0.005 A E-0.018 B C+0.066 B D \\
& +0.087 B E+0.058 C D+0.054 C E-0.036 D E,
\end{aligned}
$$

height of reinforcement $(R), \mathrm{mm}$

$$
=5.752+0.160 A-0.151 B-0.060 C+0.016 D
$$




$$
\begin{aligned}
& -0.002 E+0.084 A^{2}+0.037 B^{2}-0.0006 C^{2} \\
& +0.015 D^{2}-0.006 E^{2}+0.035 A B+0.018 A C \\
& -0.008 A D-0.048 A E-0.024 B C-0.062 B D \\
& -0.003 B E+0.012 C D-0.092 C E-0.095 D E
\end{aligned}
$$

percentage dilution $(D), \%$

$$
\begin{aligned}
= & 19.705+0.325 A+0.347 B+3.141 C-0.039 D \\
& -0.153 E-1.324 A^{2}-0.923 B^{2}-1.012 C^{2} \\
& -1.371 D^{2}-0.872 E^{2}-0.200 A B+0.346 A C \\
& +0.602 A D+0.203 A E+0.011 B C+0.465 B D \\
& +0.548 B E+0.715 C D+0.360 C E+0.137 D E .
\end{aligned}
$$

3.7. Checking the Adequacy of the Developed Models. The adequacy of the developed model was tested using the analysis of variance (ANOVA) technique. As per this technique, if the F-ratio values of the developed models do not exceed the standard tabulated values for a desired level of confidence (95\%) and the calculated R-ratio values of the developed model exceed the standard values for a desired level of confidence (95\%), then the models are said to be adequate within the confidence limit [10]. These conditions were satisfied for the developed models. The values are shown in Table 5.

\section{Artificial Neural Networks}

Artificial neural network (ANN) is biologically inspired by intelligent techniques [11]. Neural network consists of many nonlinear computational elements operating in parallel. Basically it consists of neurons; it represents our biological nervous system. The basic unit of ANN is the neuron. The neurons are connected to each other by link and are known as synapses which are associated to a weight factor. An artificial neuron receives signals from other neurons through the connection between them. Each of the connection strengths has a synaptic connection strength which is represented by a weight of that connection strength. This artificial neuron receives a weighted sum of outputs of all neurons to which it is connected. The weighted sum is then compared with the threshold for an ANN and if it exceeds this threshold ANN fires. When it fires it goes to higher excitation state and a signal is sent down to other connected neurons. The output of a typical neuron is obtained as a result of nonlinear function of weighted sum. This process is clearly shown in Figure 5.

Neural networks have many usages in the present decade. It has been successfully applied across an entire ordinary range of problems domains such as finance, medicine, energy, geology and physics where there is a problem of prediction neural network can be used successfully [12]. It is an adaptable system that can learn relationship through repeated presentation of data and is capable of generalizing a new previously unseen data. One of the most popular learning algorithms is the back propagation algorithm $[13,14]$. In this study, feedback propagation algorithm was used with a single hidden layer improved with numerical optimization technique called Levenberg-Marquard approximation algorithm (LM) [15]. The topology of architecture three-layer of feedforward back propagations network is illustrated in Figure 6.

MATLAB 7 was used for tracing the network for the prediction of clad bead geometry. Statistical mathematical model was used to compare results produced by the work. For normalizing the data, the goal is to examine the statistical distribution of values of each net input, and outputs are roughly uniform; in addition the value should be scaled to match range of input neurons [16].

This is basically in the range of 0 to 1 in practice it is found to between 01 and 9 [17]. In this paper database is normalized using (9):

$$
X_{\text {norm }}=0.1+\frac{\left(X-X_{\min }\right)}{1.25\left(X_{\max }-\left(X_{\min }\right)\right)},
$$

where $X_{\text {norm }}=$ normalized value between 0 and $1, X=$ value to be normalized, and $X_{\min }=$ minimum value in the dataset range the particular data set rage which is to be normalized. $X_{\max }=$ maximum value in the particular data set range which is to be normalized.

In this study five welding process parameters were employed as input to the network. The Levenberg-Marquardt approximation algorithm was found to be the best fit for application because it can reduce the MSE to a significantly small value and can provide better accuracy of prediction. So neural network model with feed forward back propagation algorithm and Levenberg-Marquardt approximation algorithm was trained with data collected for the experiment. Error was calculated using (10):

$$
\text { error }=\frac{(\text { actual value }- \text { predicted value }) \times 100}{\text { predicted value }}
$$

The difficulty in using the regression equation is the possibility of overfitting the data. To avoid this, the experimental data is divided into two sets: one training set and other test data set. The ANN model is created using only training data; the other test data is used to check the of behavior the ANN model created. All variables are normalized using (9). The data was randomized and portioned into two:one training and other test data,

$$
\begin{gathered}
y=\sum_{i} w_{i j} h_{i}+\theta, \\
h_{i}=\tanh \left(\sum_{j} w_{i j} x_{j}+\theta_{i}\right) .
\end{gathered}
$$

Neural network general form can be defined as a model shown above $y$ representing the output variables and $x_{j}$ the set of inputs, shown in (11) and (12). The subscript $i$ represents the hidden units shown in Figure $6, \theta$ represents bias, and $w_{j}$ represents the weights. The above equation defines the function giving output as a function of input. 
TABLE 5: Analysis of variance for testing adequacy of the model.

\begin{tabular}{|c|c|c|c|c|c|c|c|c|c|c|c|}
\hline \multirow{2}{*}{ Parameter } & \multicolumn{2}{|c|}{ 1st-order terms } & \multicolumn{2}{|c|}{ 2nd-order terms } & \multicolumn{2}{|c|}{ Lack of fit } & \multicolumn{2}{|c|}{ Error terms } & \multirow{2}{*}{$F$-ratio } & \multirow{2}{*}{$R$-ratio } & \multirow{2}{*}{ Whether model is adequate } \\
\hline & SS & $\mathrm{DF}$ & SS & DF & SS & $\mathrm{DF}$ & SS & DF & & & \\
\hline $\bar{W}$ & 36.889 & 20 & 6.233 & 11 & 3.513 & 6 & 2.721 & 5 & 1.076 & 3.390 & Adequate \\
\hline$P$ & 7.810 & 20 & 0.404 & 11 & 0.142 & 6 & 0.261 & 5 & 0.454 & 7.472 & Adequate \\
\hline$R$ & 1.921 & 20 & 0.572 & 11 & 0.444 & 6 & 0.128 & 5 & 2.885 & 3.747 & Adequate \\
\hline$D$ & 506.074 & 20 & 21.739 & 11 & 6.289 & 6 & 15.45 & 5 & 0.339 & 8.189 & Adequate \\
\hline
\end{tabular}

SS: sum of squares; DF: degree of freedom; $F$-ratio $(6,5,0.5)=3.40451 ; R$-ratio $(20,5,0.05)=3.20665$.

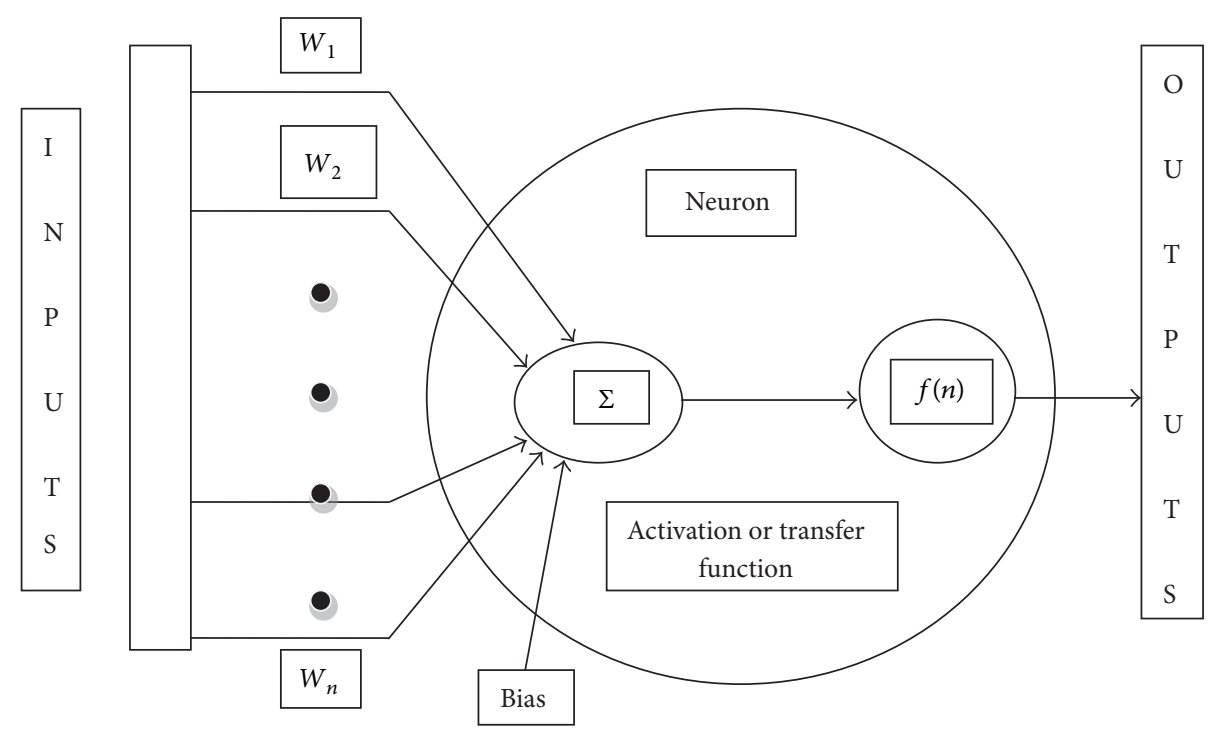

Figure 5: Model of an Artificial Neuron.

The training process involves the derivation of weights by minimization of the regularized sum of squared error.

The complexity of model is controlled by the number of hidden level values of regularization constants and are associated with each input one for biases and one for all weights connected to output.

4.1. Procedure for Prediction. The effectiveness of ANN model fully depends on the trial and error process. This study considers the factors that could be influencing the effectiveness of the model developed. In the MATLAB 7 Toolbox there are five Influencing factors listed below:

(1) network algorithm,

(2) transfer function,

(3) training function,

(4) learning function,

(5) performance function.

The ANN structure consists of three layers which are input, hidden, and output layers. It is known that ANN model is designed on trial and error basis. The trial and error is carried out by adjusting the number of layers and the number of neurons in the hidden structure. Too many neurons in hidden layer result in a waste of computer memory and computation time, while too few neurons may not provide the desired data control effect. The process is conducted using 28 randomly selected samples. Seventeen data are used for training and eleven used for testing the data. Table 4 shows randomized data with 1-11 for test data and 12-28 for training data. It is suggested that guide lines should be followed for selecting training and testing of data such as $90 \%: 10 \%$, $85 \%: 15 \%$, and $80 \%: 20 \%$ with a total of $100 \%$ combined ratio. To fit the randomized sample of 28 , preferred ratio selected is $70 \%: 30 \%$ :

(1) $(70 / 100) \times 28=19-20$ training samples,

(2) $(30 / 100) \times 28=8-9$ data testing samples.

It is necessary to normalize the quantitative variable to some standard range from 0 to 1 . The number of neurons' hidden layers should be approximately equal to $n / 2, n, 2 n$, and $2 n+1$ where $n$ is the number of input neurons. Many different ANN network algorithms have been proposed by researchers, but back propagation (BP) algorithm was found to be the best for prediction. Researchers developed the model by using feed forward BP and radial basis network algorithm, and it was found that feed forward BP gives more accurate results. Basically a feed forward network based on BP is a multilayered architecture made up of one or more hidden layers placed between input and output layers, as shown in Figure 6. Transfer function, training function, learning 


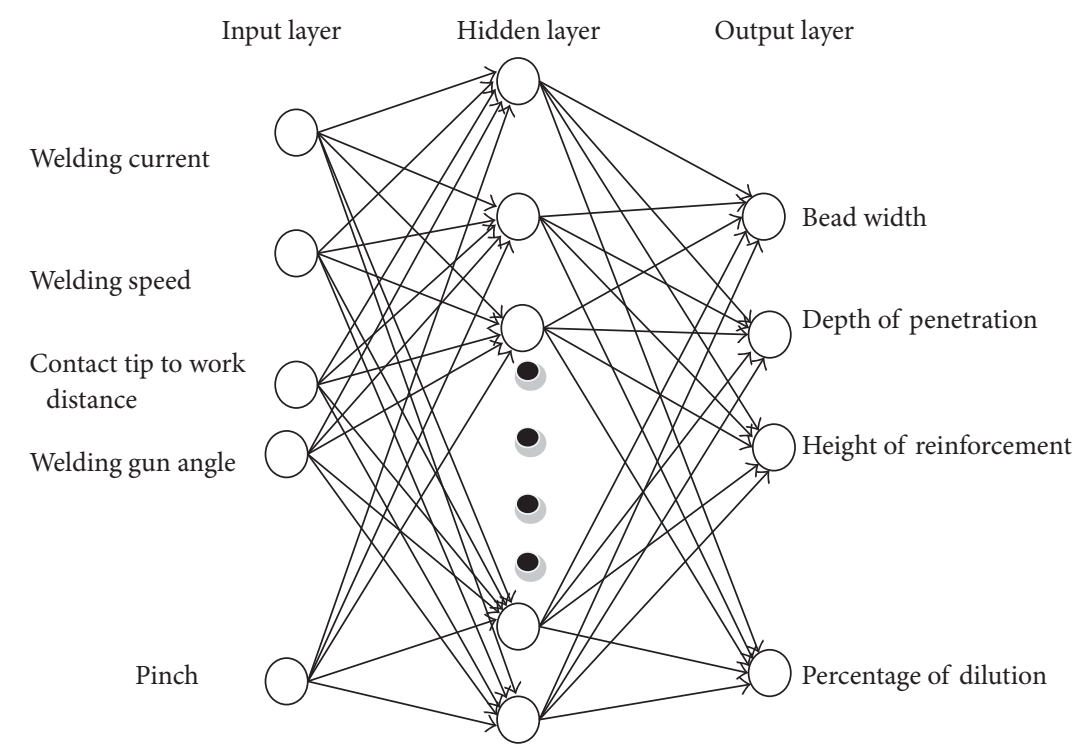

FIGURE 6: Neural network architecture.

function, and performance function used in this study are logsig, traindgm, traingdx, and MSE.

4.2. Determination of the Best ANN Model. In order to determine the best network structure of ANN prediction model, the randomized data set is divided into two sets: one training data set which is used for the prediction of the model, and the other test data which is used to validate the model. Seventeen data were used for training set and eleven data for test set. This to avoid the over fitting of the data. An ANN model is created and trained using MATLAB 7 ANN Toolbox. The lowest MSE obtained is for twelve hidden neurons. So a network structure is 5-12-4: five input neurons, twelve hidden neurons, and four output neurons. Then the test data is validated against the ANN model created; the results are shown in Table 6. Training data is shown in Table 7.

\section{Genetic Algorithm Optimization}

Genetic algorithm is meta heuristic searching techniques, which mimics the principles of evaluation and natural genetics. These are guided by the random search which scans through entire sample space and therefore provide a reasonable solution. It was introduced by Holland (1975). It is also considered as a heuristic technique inspired by natural biological evolution process comprising selection, cross over mutation, and so forth.

In biological population genetic information is stored in the form of binary strings. The basic processes which affect the binary strings makeup in natural evolution are a selection, a crossover of genetic information between reproducing parents, a mutation of genetic information, and an elitist strategy that keeps the best individual to next generation. The main operations of GA are characterized as follows.

Selection is a method that randomly picks up chromosomes out of the population according to the evolution
Crossover point

Crossover point

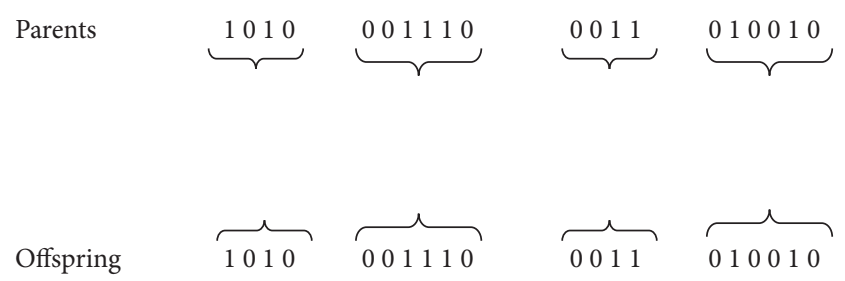

FIGURE 7: Single-Point cross over.

function. The higher the fitness function is, the more chance of an individual has to be selected. The selection pressure is defined as the degree to which the better individuals are favoured.

It takes two individuals and puts their chromosome strings at some randomly chosen position to produce two head segments and two tail segments. The tail segments are then supposed to produce two new full length chromosomes as shown in Figure 7. Two off spring, each inherits some genes from each parent. This is known as single-point cross over. Cross over is not usually applied to all pairs of individuals selected or mating. A random choice is made where likelihood of cross over appeared typically between 6 and 10. If cross over is not applied, off spring are produced simply by duplicating the parents. This gives each individual a chance of passing on its genes without duplication of cross over.

Mutation is applied to each child individually after cross over. It randomly alters each gene with small probability (typically .001) Table 8 shows the fifth gene of a chromosome being mutated. Traditional view is that cross over is more important than the two techniques for rapidly exploring a search space. Mutation provides a small amount of random 
TABLE 6: Comparison of actual and predicted values of the clad bead parameters using neural network data (test).

\begin{tabular}{lccccccccccccc}
\hline Trial no. & \multicolumn{3}{c}{ Actual bead parameters } & \multicolumn{4}{c}{ Predicted bead parameters } & \multicolumn{3}{c}{ Error } \\
& $\begin{array}{lcccccccccccc}D \\
(\mathrm{~mm})\end{array}$ & $\begin{array}{c}P \\
(\mathrm{~mm})\end{array}$ & $\begin{array}{c}R \\
(\mathrm{~mm})\end{array}$ & $\begin{array}{c}D \\
(\%)\end{array}$ & $\begin{array}{c}W \\
(\mathrm{~mm})\end{array}$ & $\begin{array}{c}P \\
(\mathrm{~mm})\end{array}$ & $\begin{array}{c}R \\
(\mathrm{~mm})\end{array}$ & $\begin{array}{c}D \\
(\%)\end{array}$ & $\begin{array}{c}W \\
(\mathrm{~mm})\end{array}$ & $\begin{array}{c}P \\
(\mathrm{~mm})\end{array}$ & $\begin{array}{c}R \\
(\mathrm{~mm})\end{array}$ & $\begin{array}{c}D \\
(\%)\end{array}$ \\
\hline 1 & 6.9743 & 1.6735 & 6.0262 & 10.721 & 6.1945 & 1.85 & 5.9611 & 12.367 & 0.7798 & -0.177 & 0.0651 & -1.646 \\
2 & 7.6549 & 1.9715 & 5.8873 & 12.167 & 7.1815 & 2.1507 & 6.5553 & 10.268 & 0.4734 & -0.179 & -0.668 & 1.899 \\
3 & 6.3456 & 1.6986 & 5.4519 & 12.746 & 7.4954 & 1.5339 & 5.4923 & 9.3808 & -1.15 & 0.1647 & -0.04 & 3.3652 \\
4 & 7.7635 & 1.7396 & 6.0684 & 10.611 & 6.4936 & 1.854 & 6.5573 & 9.4799 & 1.2699 & -0.114 & -0.489 & 1.1311 \\
5 & 7.2683 & 2.443 & 5.7206 & 16.673 & 7.3354 & 2.6576 & 5.5657 & 19.104 & -0.067 & -0.215 & 0.1549 & -2.431 \\
6 & 9.4383 & 2.4905 & 5.9169 & 15.967 & 7.6066 & 2.1045 & 6.4342 & 18.49 & 1.8317 & 0.386 & -0.517 & -2.523 \\
7 & 6.0823 & 2.4672 & 5.492 & 16.589 & 8.0417 & 2.1722 & 5.5126 & 16.874 & -1.959 & 0.295 & -0.021 & -0.285 \\
8 & 8.4666 & 2.0737 & 5.9467 & 14.985 & 8.3236 & 2.2349 & 5.9031 & 16.972 & 0.143 & -0.161 & 0.0436 & -1.987 \\
9 & 6.3029 & 1.5809 & 5.9059 & 10.275 & 8.2381 & 1.7955 & 5.6022 & 11.219 & -1.935 & -0.215 & 0.3037 & -0.944 \\
10 & 7.0136 & 1.5662 & 5.9833 & 9.7073 & 7.5899 & 2.4579 & 6.542 & 13.415 & -0.576 & -0.892 & -0.559 & -3.708 \\
11 & 6.2956 & 1.586 & 5.5105 & 11.117 & 7.7318 & 1.7647 & 5.8676 & 10.71 & -1.436 & -0.179 & -0.357 & 0.407 \\
\hline
\end{tabular}

TABLE 7: Comparison of actual and predicted values of the clad bead parameters using neural network data (training).

\begin{tabular}{|c|c|c|c|c|c|c|c|c|c|c|c|c|}
\hline \multirow{2}{*}{ Trial no. } & \multicolumn{4}{|c|}{ Actual bead parameters } & \multicolumn{4}{|c|}{ Predicted bead parameters } & \multicolumn{4}{|c|}{ Error } \\
\hline & $\begin{array}{c}W \\
(\mathrm{~mm})\end{array}$ & $\begin{array}{c}P \\
(\mathrm{~mm})\end{array}$ & $\begin{array}{c}R \\
(\mathrm{~mm})\end{array}$ & $\begin{array}{c}D \\
(\%)\end{array}$ & $\begin{array}{c}W \\
(\mathrm{~mm})\end{array}$ & $\begin{array}{c}P \\
(\mathrm{~mm})\end{array}$ & $\begin{array}{c}R \\
(\mathrm{~mm})\end{array}$ & $\begin{array}{c}D \\
(\%)\end{array}$ & $\begin{array}{c}W \\
(\mathrm{~mm})\end{array}$ & $\begin{array}{c}P \\
(\mathrm{~mm})\end{array}$ & $\begin{array}{c}R \\
(\mathrm{~mm})\end{array}$ & $\begin{array}{l}D \\
(\%)\end{array}$ \\
\hline & 7.741 & 1.8466 & 5.8752 & 11.4273 & 7.335 & 2.0986 & 6.0792 & 10.8222 & 0.406 & -0.252 & -0.204 & 0.6051 \\
\hline & 7.3231 & 2.16475 & 5.72095 & 15.29097 & 6.8214 & 2.0617 & 5.6946 & 14.9379 & 0.5017 & 0.10305 & 0.02635 & 0.35307 \\
\hline 3 & 9.6171 & 2.69495 & 6.37445 & 18.54077 & 9.3713 & 2.8982 & 6.4084 & 17.4578 & 0.2458 & 0.20325 & -0.0339 & 1.08297 \\
\hline 4 & 6.6335 & 2.3089 & 5.554 & 17.23138 & 7.4306 & 2.2927 & 5.6232 & 15.7908 & -0.7971 & 0.0162 & -0.0692 & 1.44058 \\
\hline 5 & 10.514 & 2.7298 & 5.4645 & 20.8755 & 7.8991 & 2.5154 & 5.8078 & 18.0664 & 2.6149 & 0.2144 & -0.3433 & 2.8091 \\
\hline 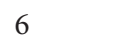 & & 1.99045 & 5.80585 & 13.65762 & 6.5761 & 1.9158 & 5.7867 & 14.2039 & -0.0204 & 0.07465 & 0.01915 & -0.5462 \\
\hline 7 & & 2.5737 & 6.65505 & 15.74121 & 7.393 & 2.7191 & 6.7112 & 14.7525 & & -0.1454 & -0.0561 & 0.98871 \\
\hline 8 & 7.5886 & 2.50455 & 6.4069 & 15.77816 & 7.5943 & 2.4317 & 6.3834 & 15.9881 & -0.0057 & 0.07285 & 0.0235 & -0.2099 \\
\hline 9 & 7.5014 & 2.1842 & 5.6782 & 16.82349 & 7.4652 & 2.2814 & 5.7674 & 16.5744 & 0.0362 & -0.0972 & -0.0892 & 0.24909 \\
\hline 10 & 6.1421 & 1.3752 & 6.0976 & 8.941799 & 5.6583 & 1.44 & 6.2054 & 9.3753 & 0.4838 & -0.0648 & -0.1078 & -0.4335 \\
\hline 11 & 8.5647 & 3.18536 & 5.63655 & 22.94721 & 9.9724 & 2.962 & 5.5227 & 18.9566 & -1.4077 & 0.22336 & 0.11385 & 3.99061 \\
\hline 12 & 7.9575 & 2.2018 & 5.8281 & 15.74941 & 9.0693 & 2.6919 & 6.2337 & 17.5548 & -1.1118 & -0.4901 & -0.4056 & -1.8053 \\
\hline 13 & 7.7085 & 1.85885 & 6.07515 & 13.27285 & 6.7699 & 1.7807 & 6.109 & 12.8584 & 0.9386 & 0.07815 & -0.0338 & 0.41445 \\
\hline 14 & 7.8365 & 2.3577 & 5.74915 & 16.63287 & 8.5364 & 2.9431 & 6.6735 & 15.9653 & -0.6999 & -0.5854 & -0.9243 & 0.66757 \\
\hline 15 & 8.2082 & 2.3658 & 5.99005 & 16.38043 & 8.0083 & 2.371 & 6.0186 & 16.3701 & 0.1999 & -0.0052 & -0.0285 & 0.01033 \\
\hline 16 & 7.9371 & 2.1362 & 6.0153 & 15.18374 & 7.9441 & 2.1197 & 6.01 & 15.3735 & -0.007 & 0.0165 & 0.0053 & -0.1897 \\
\hline 17 & 8.4731 & 2.17145 & 5.69895 & 14.82758 & 8.6735 & 2.5165 & 5.4985 & 15.2875 & -0.2001 & -0.3450 & 0.2031 & -0.4599 \\
\hline
\end{tabular}

TABLE 8: Single mutations.

\begin{tabular}{lllllllllll}
\hline Offspring & 1 & 0 & 1 & 0 & 0 & 1 & 0 & 0 & 1 & 0 \\
Mutual offspring & 1 & 0 & 1 & 0 & 1 & 1 & 0 & 0 & 1 & 0 \\
\hline
\end{tabular}

search and help to ensure that no point in the space has zero probability of being examined. Two individuals can reproduce to give two off spring as shown in Figure 7.

The fitness function is an exponential function of one variable with a maximum $x=.2$ which is coded as a 10-bit binary number in Table 9. Table 9 shows two parents as off spring they produce crossed over after second bit.
TABLE 9: Details of individual.

\begin{tabular}{lccc}
\hline Individual & $X$ & Fitness & Chromosome \\
\hline Parent I & 0.08 & 0.05 & 0001010010 \\
Parent II & 0.73 & 0.000002 & 1011101011 \\
Offspring I & 0.23 & 0.47 & 0011101011 \\
Offspring II & 0.58 & 0.00007 & 1001010010 \\
\hline
\end{tabular}

If genetic algorithm has been correctly implemented, the population will evolve over successive generation so that fitness of the best and the average individual in each generation increases towards the global optimum. Convergence is the progression towards increasing uniformity. A gene is said to 


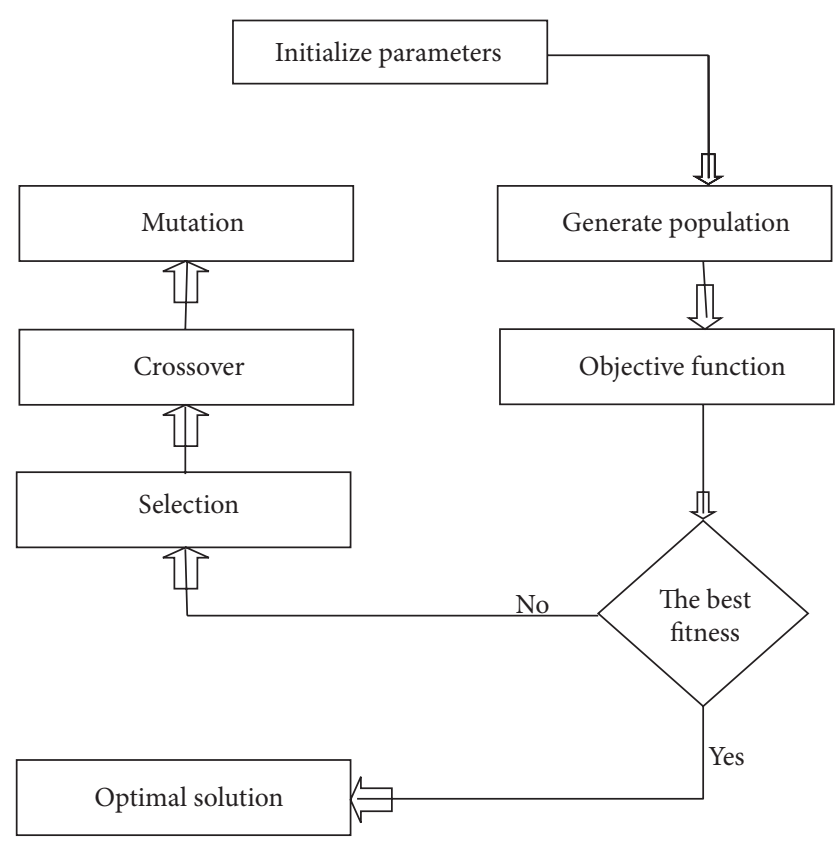

FIgure 8: Traditional Genetic Algorithm.

TABLE 10: GA search ranges.

\begin{tabular}{lc}
\hline Parameters & Range \\
\hline Welding current $(I)$ & $200-300 \mathrm{amp}$ \\
Welding speed $(S)$ & $150-182 \mathrm{~mm} / \mathrm{min}$ \\
Contact tip to work distance $(N)$ & $10-26 \mathrm{~mm}$ \\
Welding gun angle $(T)$ & $70-110 \mathrm{deg}$ \\
Pinch $($ Ac) & $-10-10$ \\
\hline
\end{tabular}

TABLE 11: For GA computation.

\begin{tabular}{lc}
\hline Population type & Double vector \\
Population size & 30 \\
Fitness scaling function & Rank \\
Selection function & Roulette \\
Reproduction elite count & 2 \\
Cross over rate & 100 \\
Cross over function & Intermediate \\
Mutation function & Uniform \\
Mutation rate & $1 \%$ \\
Number of generation & 52 \\
Migration & Forward \\
\hline
\end{tabular}

have converged when $95 \%$ of the population shares the same value (Dejony 1975). The population is said to have converged when all of the genes have converged. Traditional genetic algorithm is shown in Figure 8.

\section{Optimization of Clad Bead Geometry Using GA}

The experimental data related to welding current $(I)$, welding speed $(S)$, welding gun angle $(T)$, contact tip to work distance $(N)$, and pinch (Ac) is used in the experiments conducted.

The aim of the study is to find for optimum adjustments for welding current $(I)$, welding speed $(S)$, welding gun angle $(T)$, contact tip to work distance $(N)$, and pinch (Ac) in a GMAW cladding process. Table 10 shows the options used for study. Table 11 shows GA computation.

The objective function selected for optimizing was the percentage of dilution. The response variables bead width $(W)$, Penetration $(P)$, reinforcement $(R)$, and dilution $(D)$ were given as constraints in their equation. The constrained nonlinear optimiation is mathematically stated as follows:

$$
\begin{aligned}
& \text { minimize } f(x) \\
& \text { subject to } f(X(1), X(2), X(3), X(4), X(5))<0 .
\end{aligned}
$$

Genetic algorithms are nowadays a popular tool in optimizing because GA uses only the values of objective function. The derivatives are not used in the procedure. Secondly the objective function value corresponding to a design vector plays the role of fitness in natural genetics. The aim of the study is to find the optimum adjustments for welding current, welding speed, pinch, welding angle, and contact to tip distance. Objective function selected for optimization was the percentage of dilution. The process parameters and their notations used in writing the program in MATLAB 7 software are given below:

$$
\begin{aligned}
& X(1)=A=\text { welding current }(I) \text { in amp, } \\
& X(2)=B=\text { welding Speed }(S) \text { in } \mathrm{mm} / \mathrm{min}, \\
& X(3)=C=\text { contact to work piece distance }(N) \text { in } \\
& \mathrm{mm}, \\
& X(4)=D=\text { Welding gun angle }(T) \text { in degree, } \\
& X(5)=E=\text { pinch }(\mathrm{Ac}),
\end{aligned}
$$

Objective function for percentage of dilution which must be minimized was derived from (5)-(8). The constants of welding parameters are given in Table 2

subjected to bounds

$$
\begin{aligned}
& 200 \leq X(1) \leq 300, \\
& 150 \leq X(2) \leq 182, \\
& 10 \leq X(3) \leq 26, \\
& 70 \leq X(4) \leq 110, \\
& -10 \leq X(5) \leq 10 .
\end{aligned}
$$

6.1. Objective Function. Consider the following:

$$
\begin{aligned}
f(x)= & 19.75+0.325 * x(1)+0.347 * x(2) \\
& +3.141 * x(3)-0.039 * x(4)-0.153 * x(5)
\end{aligned}
$$




$$
\begin{aligned}
& -1.324 * x(1)^{2}-0.923 * x(2)^{2}-1.012 * x(3)^{2} \\
& -1.371 * x(4)^{2}-0.872 * x(5)^{2} 0.200 * x(1) \\
& * x(2)+0.346 * x(1) * x(3)+0.602 * x(1) \\
& * x(4)+0.203 * x(1) * x(5)+0.011 * x(2) \\
& * x(3)+0.465 * x(2) * x(4)+0.548 * x(2) \\
& * x(5)+0.715 * x(3) * x(4)+0.360 * x(3) \\
& * x(5)+0.137 * x(4) * x(5) .
\end{aligned}
$$

6.2. Constraint Equations. Consider

$$
\begin{aligned}
W=( & 823+0.701 * x(1)+0.388 * x(2) \\
& +0.587 * x(3)+0.040 * x(4)+0.088 * x(5) \\
& -0.423 * x(1)^{2}-0.291 * x(2)^{2}-0.338 * x(3)^{2} \\
& -0.219 * x(4)^{2} 0.171 * x(5)^{2}+0.205 * x(1) * x(2) \\
& +0.405 * x(1) * x(3)+0.105 * x(1) * x(4) \\
& +0.070 * x(1) * x(5) 0.134 * x(2) * x(3) \\
& +0.2225 * x(2) * x(4)+0.098 * x(2) * x(5) \\
& +0.26 * x(3) * x(4)+0.086 * x(3) * x(5) \\
& +0.12 * x(4) * x(5))-3
\end{aligned}
$$

(clad bead width $(W)$ mm lower limit). Consider

$$
\begin{aligned}
P=( & .735+0.098 * x(1)-0.032 * x(2) \\
& +0.389 * x(3)-0.032 * x(4)-0.008 * x(5) \\
& -0.124 * x(1)^{2}-0.109 * x(2)^{2}-0.125 * x(3)^{2} \\
& -0.187 * x(4)^{2}-0.104 * x(5)^{2}-0.33 * x(1) * x(2) \\
& +0.001 * x(1) * x(3)+0.075 * x(1) * x(4) \\
& +0.005 * x(1) * x(5)-0.018 * x(2) * x(3) \\
& +0.066 * x(2) * x(4)+0.087 * x(2) * x(5) \\
& +0.058 * x(3) * x(4)+0.054 * x(3) * x(5) \\
& -0.036 * x(4) * x(5))-3
\end{aligned}
$$

(depth of penetration $(P)$ upper limit). Consider

$$
\begin{aligned}
P=( & 2.735+0.098 * x(1)-0.032 * x(2)+0.389 * x(3) \\
& -0.032 * x(4)-0.008 * x(5)-0.124 * x(1)^{2} \\
& -0.109 * x(2)^{2}-0.125 * x(3)^{2}-0.187 * x(4)^{2} \\
& -0.104 * x(5)^{2}-0.33 * x(1) * x(2)+0.001 * x(1) \\
& * x(3)+0.075 * x(1) * x(4)+0.005 * x(1) \\
& * x(5)-0.018 * x(2) * x(3)+0.066 * x(2) \\
& * x(4)+0.087 * x(2) * x(5)+0.058 * x(3) * x(4) \\
& +0.054 * x(3) * x(5)-0.036 * x(4) * x(5))+2
\end{aligned}
$$

(depth of penetration $(P)$ lower limit). Consider

$$
\begin{aligned}
W=( & 8.923+0.701 * x(1)+0.388 * x(2) \\
& +0.587 * x(3)+0.040 * x(4)+0.088 * x(5) \\
& -0.423 * x(1)^{2}-0.291 * x(2)^{2}-0.338 * x(3)^{2} 0.219 \\
& * x(4)^{2} 0.171 * x(5)^{2}+0.205 * x(1) * x(2)+0.405 \\
& * x(1) * x(3)+0.105 * x(1) * x(4)+0.070 * x(1) \\
& * x(5) 0.134 * x(2) * x(3)+0.225 * x(2) * x(4) \\
& +0.098 * x(2) * x(5)+0.26 * x(3) * x(4) \\
& +0.086 * x(3) * x(5)+0.012 * x(4) * x(5))-10
\end{aligned}
$$

(clad bead width $(W)$ upper limit). Consider

$$
\begin{aligned}
& R=(5.752+0.160 * x(1)-0.151 * x(2)-0.060 \\
& * x(3)+0.016 * x(4)-0.002 * x(5)+0.084 \\
& * x(1)^{2}+0.037 * x(2)^{2}-0.0006 * x(3)^{2}+0.015 \\
& * x(4)^{2}-0.006 * x(5)^{2}+0.035 * x(1) \\
& * x(2)+0.018 * x(1) * x(3)-0.008 * x(1) \\
& * x(4)-0.048 * x(1) * x(5)-0.024 * x(2) \\
& * x(3)-0.062 * x(2) * x(4)-0.003 * x(2) \\
& * x(5)+0.012 * x(3) * x(4)-0.092 * x(3) \\
&* x(5)-0.095 * x(4) * x(5))-6
\end{aligned}
$$


(height of reinforcement $(R)$ lower limit). Consider

$$
\begin{aligned}
R=( & 552+0.160 * x(1)-0.151 * x(2)-0.060 \\
& * x(3)+0.016 * x(4)-0.002 * x(5)+0.084 \\
& * x(1)^{2}+0.037 * x(2)^{2}-0.0006 * x(3)^{2}+0.015 \\
& * x(4)^{2}-0.006 * x(5)^{2}+0.035 * x(1) * x(2) \\
& +0.018 * x(1) * x(3)-0.008 * x(1) * x(4) \\
& -0.048 * x(1) * x(5)-0.024 * x(2) * x(3) \\
& -0.062 * x(2) * x(4)-0.003 * x(2) * x(5) \\
& +0.012 * x(3) * x(4)-0.092 * x(3) * x(5) \\
& -0.095 * x(4) * x(5))+6
\end{aligned}
$$

(heights of reinforcement $(R)$ upper limit),

$$
\begin{gathered}
f(x)-23.6334 \\
8.94441799-f(x) .
\end{gathered}
$$

(dilution upper and lower limits),

$$
\begin{aligned}
& x(1), x(2), x(3), x(4), x(5) \leq 2, \\
& x(1), x(2), x(3), x(4), x(5) \geq-2 .
\end{aligned}
$$

MATLAB program in GA and GA function were used for optimizing the problem. The program was written in GA and constraint bounds were applied. The minimum percentage of dilution obtained from the results was while obtained running the GA tool. The minimum percentage of dilution obtained is 10.396. the value of process parameters obtained is $I=240 \mathrm{amp}, S=157 \mathrm{~mm} / \mathrm{min}, N=13 \mathrm{~mm}, T=87$ degrees, and $A c=4.5$. The fitness function is shown in Figure 9 .

\section{Methodology of Integrated ANN-GA}

The methodology applied in this study involves six cases [18]. They are experimental data, regression modeling, ANN single-based modeling, GA-single based optimization, integrated ANN-GA-type-A-based optimization and integrated ANN-GA-type-B-based optimization. The objectives of type $A$ and type $B$ are as follows.

(1) To estimate the minimum values of cladding parameters compared to the performance values of the experimental data, regression modeling, and ANN single-based modeling.

(2) To estimate the optimal process parameters values that have been within the range of minimum and maximum coded values for process parameters of experimental design that are used for experimental trial.

(3) To estimate the optimal solution of the process parameters within the small number of iterations compared to the optimal solution of the process parameters with single-based GA optimization.

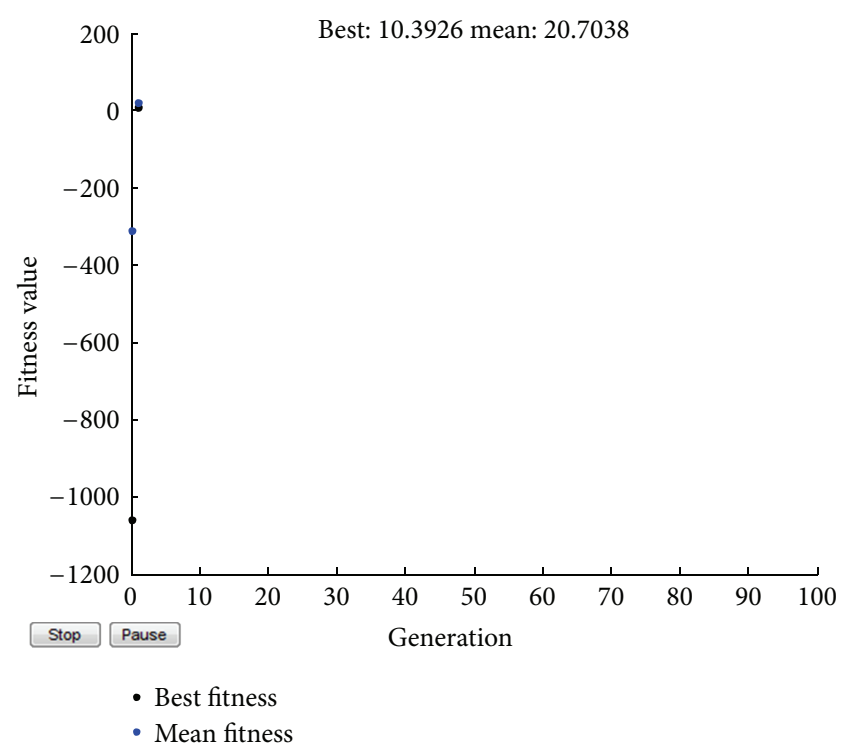

FIgURE 9: Fitness value of GA function.

The steps in order to implement the integrated ANN-GA type $A$ and integrated ANN-GA type-B in fulfilling the three objectives are as follows.

(1) In the experimental data module the values of dilution for different combinations of process parameters are used for modeling.

(2) In the regression modeling schedule model was developed using cladding process parameters. A multilinear regression analysis was performed to predict dilution, and a governing equation was constructed [19].

(3) A predicted model was developed using ANN. The percentage of error was calculated between predicted and actual values.

(4) In the single-based GA optimization, the predicted equation of the regression model would become the objective function. The minimum and maximum coded values of the process parameters of the experimental design would define the boundaries for minimum and maximum values of the optimal solution.

(5) In the integrated ANN-GA type A module which was the first integration system proposed in this study. Similar to GA single-based optimization process, the predicted equation of the regression would become the objective function. The integration system possesses the optimal process parameters value of the single based GA optimization process combined with the process parameters of ANN system would define the boundaries for minimum and maximum values for optimal solution.

(6) In the integrated ANN-GA type B system which is the second integration system proposed in this study. Similar to type A, the predicted regression 
TABLE 12: Conditions to define limitation constraint bounds of integrated ANN-GA.

\begin{tabular}{|c|c|c|}
\hline \multirow{2}{*}{ Condition } & \multicolumn{2}{|c|}{ Decision } \\
\hline & Lower bound & Upper bound \\
\hline (1) $\left(\mathrm{Opt}_{\mathrm{ANN}}\right)<\left(\mathrm{Opt}_{\mathrm{GA}}\right)$ & $\mathrm{Opt}_{\mathrm{ANN}}$ & $\mathrm{Opt}_{\mathrm{GA}}$ \\
\hline (2) $\left(\mathrm{Opt}_{\mathrm{ANN}}\right)>\left(\mathrm{Opt}_{\mathrm{GA}}\right)$ & $\mathrm{Opt}_{\mathrm{GA}}$ & $\mathrm{Opt}_{\mathrm{ANN}}$ \\
\hline (3) $\left(\mathrm{Opt}_{\mathrm{ANN}}\right)=\left(\mathrm{Opt}_{\mathrm{GA}}\right)$ & $\begin{array}{l}\text { Nearest lower } \\
\text { bound of the } \\
\text { coded value of } \\
\text { experimental } \\
\text { design }\end{array}$ & $\begin{array}{l}\text { Nearest upper } \\
\text { bound of } \\
\text { the coded value } \\
\text { of experimental } \\
\text { design }\end{array}$ \\
\hline
\end{tabular}

equation would become the objective function and the optimal process parameters value of single-based GA optimization combined with process parameters value of the ANN model would define the boundaries for the minimum and maximum values of the optimal solution. This integration system proposes the process parameters values of the best ANN model to define the initial point for optimization solution. This process is shown in Figure 10 [20].

7.1. Integrated ANN-GA Type A optimization Solution. The strategy of this study in implementing integrated ANN-GA type $\mathrm{A}$ is implemented by proposing the optimal process parameters value of the GA combined with the nonoptimal process parameters value of the ANN model to define the boundaries for the minimum and maximum value for optimization solution [21]. As given in Table 4, the nonoptimal process parameters values that yield to the minimum prediction value of the ANN model for dilution are $D \%=$ 9.353, $I=250 \mathrm{amp}, S=166 \mathrm{~mm} / \mathrm{min}, N=10 \mathrm{~mm}, T=$ 10 degree, and $A c=0$. As given in Figure 9, the values of optimal process parameters from GA are $I=240 \mathrm{amp}$, $S=157 \mathrm{~mm} / \mathrm{min}, N=13 \mathrm{~mm} . T=87$ degrees, and $\mathrm{Ac}=4.5$.

Three conditions could be stated for the nonoptimal process parameters values of the ANN model (OptANN) and optimal process parameters values of the GA (OptGA) as classified in Table 12.

By using the conditions stated in Table 12, the decision to define the limitation constraint bound values of the optimization solution is given. Fulfilling the above conditions, (21)(28) are formulated to define the limitation constraint bounds for welding current, welding speed, contact tip to work distance, welding angle, and pinch as process parameters, respectively. The limitation constraint bound for each process parameter is stated as follows [22]:

$$
\begin{gathered}
240 \leq I \leq 250, \\
157 \leq S \leq 166, \\
10 \leq N \leq 13, \\
87 \leq T \leq 90, \\
0 \leq \text { Ac } \leq 4.5 .
\end{gathered}
$$

As per Figure 10 the optimal process parameters values that lead to the minimum cladding performance of the GA are proposed to define initial points for the integrated ANN-GA-type A to search for the optimal solution [23]. By considering the optimal solution of process parameters of the GA shown in Figure 9, the equations for the initial point of optimization solution for integrated ANN-GA type A are given in (22), (30), (31), (32), and (33) as follows:

$$
\begin{aligned}
& \text { initial point of } I=240, \\
& \text { initial point of } S=157, \\
& \text { initial point of } N=10, \\
& \text { initial point of } T=87, \\
& \text { initial point of } A c=0 .
\end{aligned}
$$

By using the objective function formulated in (12), the limitation constraint bounds of process parameters formulated in (21), (25), (26), (27), and (28), the initial points formulated in (22), (30), (31), (32), and (33), and the same setting of parameters applied in GA given in Table 12, results of the integrated ANN-GA type A by using MATLAB 7 Optimization Toolbox fitness function are obtained as shown in Figure 11 [19].

It can be seen that the set values of optimal process parameters that lead to the minimum dilution $D=9.7467 \%$ are $I=274 \mathrm{amp}, S=152 \mathrm{~mm} / \mathrm{min}, N=12 \mathrm{~mm}, T=73$ degrees, and $\mathrm{Ac}=1.4$.

7.2. Integrated ANN-GA Type B Optimization Solution. Similar to integrated ANN-GA type A approach, the objective function formulated in (12), The basic difference to the integrated ANN-GA type A approach, the nonoptimal process parameters values that led to minimum WELDING performance of the best ANN model shown in Figure 11 is proposed to define initial points for the integrated ANNGA Type B is to search the optimal solution. Therefore, the equation for the initial point for integrated ANN-GA type B could be given in (23) to (25) as follows:

$$
\begin{aligned}
& \text { initial point of } I=250, \\
& \text { initial point of } S=166 \text {, } \\
& \text { initial point of } N=10, \\
& \text { initial point of } T=90, \\
& \text { initial point of } A c=0 .
\end{aligned}
$$

The results of the integrated ANN-GA type B by using MATLAB Optimization Toolbox fitness function are shown in Figure 12.

From Figure 8 it can be seen that the set values of optimal process parameters that lead to the minimum value of dilution $D=9.7467$ are $I=212 \mathrm{amp} S=138 \mathrm{~mm} / \mathrm{min}$, $N=16 \mathrm{~mm}, T=78$ degrees, and $\mathrm{Ac}=1.5$. 


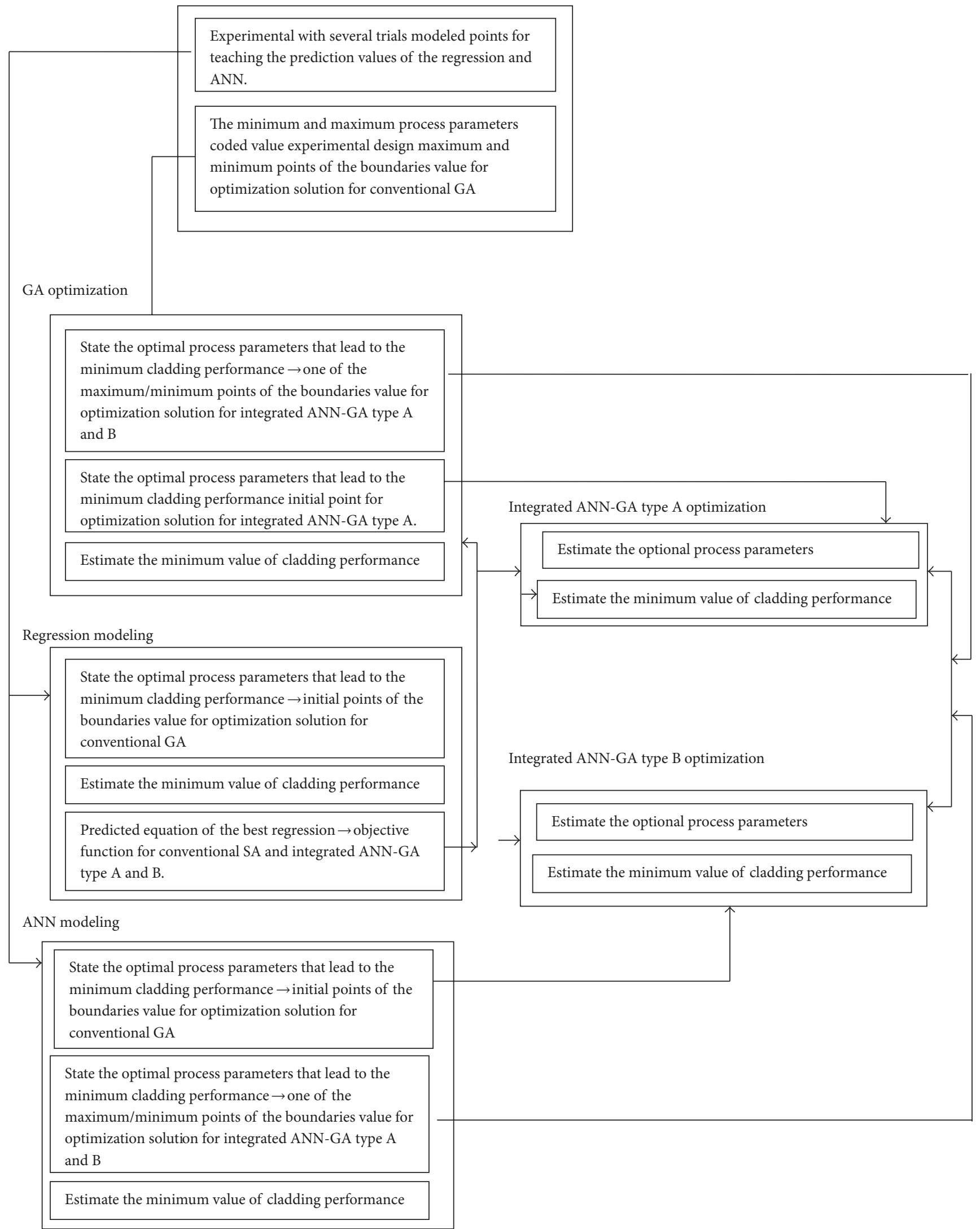

FIGURE 10: Integrated ANN-GA optimization procedure. 


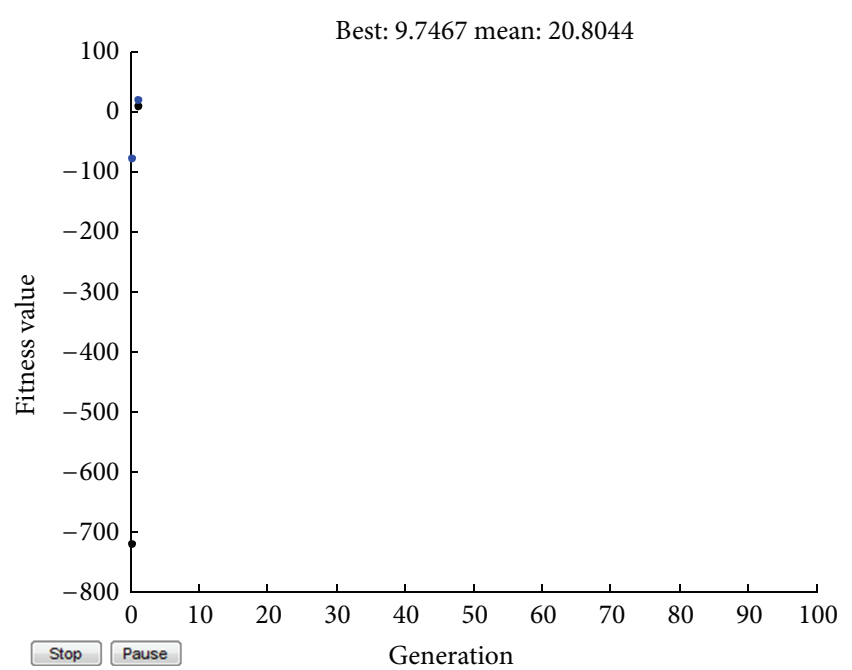

- Best fitness

- Mean fitness

FIgURE 11: Fitness value of ANN-GA Type-A.

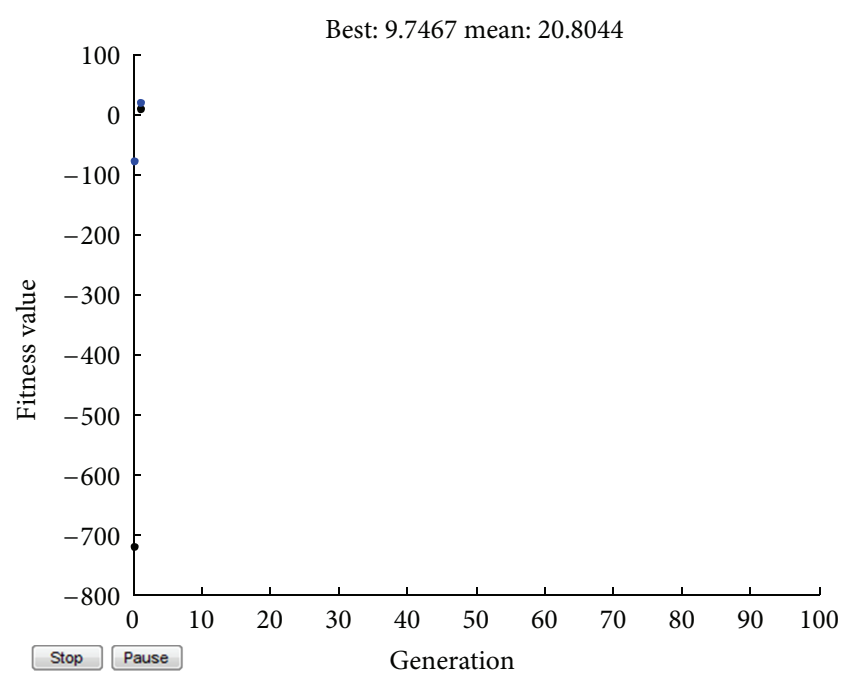

- Best fitness

- Mean fitness

FIgURE 12: Fitness value of ANN-GA Type-B.

\subsection{ANN-GA Type A. Consider}

$$
\begin{aligned}
D= & 19.75+0.325 * x(1)+0.347 * x(2)+3.141 \\
& * x(3)-0.039 * x(4)-0.153 * x(5)-1.324 \\
& * x(1)^{2}-0.923 * x(2)^{2}-1.012 * x(3)^{2}-1.371 \\
& * x(4)^{2}-0.872 * x(5)^{2}-0.200 * x(1) * x(2)+0.346 \\
& * x(1) * x(3)+0.602 * x(1) * x(4)+0.203 * x(1) \\
& * x(5)+0.011 * x(2) * x(3)+0.465 * x(2) * x(4)
\end{aligned}
$$

$$
\begin{aligned}
& +0.548 * x(2) * x(5)+0.715 * x(3) * x(4) \\
& +0.360 * x(3) * x(5)+0.137 * x(4) * x(5)
\end{aligned}
$$

optimum dilution obtained is 9.9532 .

\subsection{ANN-GA Type B}

$$
\begin{aligned}
D= & 19.75+0.325 * x(1)+0.347 * x(2)+3.141 \\
& * x(3)-0.039 * x(4)-0.153 * x(5)-1.324 \\
& * x(1)^{2}-0.923 * x(2)^{2}-1.012 * x(3)^{2} \\
& -1.371 * x(4)^{2}-0.872 * x(5)^{2}-0.200 \\
& * x(1) * x(2)+0.346 * x(1) * x(3)+0.602 * x(1) \\
& * x(4)+0.203 * x(1) * x(5)+0.011 * x(2) \\
& * x(3)+0.465 * x(2) * x(4)+0.548 * x(2) \\
& * x(5)+0.715 * x(3) * x(4)+0.360 * x(3) \\
& * x(5)+0.137 * x(4) * x(5)
\end{aligned}
$$

optimum dilution obtained is 9.7895 .

\section{Validation of the Integrated ANN-GA Results}

Theoretically, to validate the results of the proposed approach of this study, the optimal process parameters values of the integrated ANN-GA will be transferred into the regression model equation. Equation (12) is taken as the objective function of the optimization solution. With $I$ being an optimal solution of the welding current. $S$ is an optimal solution of the welding speed; $N$ is an optimal solution of contact to work distance. $T$ is an optimal solution of the welding gun angle, and Ac is an optimal solution of the pinch of integrated ANNGA. This study discusses the calculation for validating the result of integrated ANN-GA type A and integrated ANNGA type B.

\section{Evaluation of the Integrated ANN-GA Results}

In this study, discussion is carried out to highlight all the objectives of the study and is separated into three parts which are evaluation of the minimum dilution value, evaluation of the optimal process parameters, and evaluation of the number of iteration of the integrated ANN-GA results.

9.1. First Objective: Evaluation of the Minimum Dilution Value. Figures 11 and 12 show the minimum value of dilution for both integration systems, integrated ANN-GA type A and ANNGA type B, is 9.7467. For fulfilling the first objective of this 
study, evaluation against the minimum dilution is 10.3926 by GA.

9.2. Experimental Data versus Integrated ANN-GA. Optimum dilution should be between 8 and 15\% from a previous study. This objective is fulfilled in this case.

9.3. Regression versus Integrated ANN. The minimum predicted dilution value of the regression model is 9.875. Therefore, with dilution is 9.7467 , It can be concluded that both integration systems have given the more minimum result of the dilution compared to regression model. Consequently, integrated ANN-GA type A and integrated ANN-GA type B have reduced the value of dilution and optimized it between 8 to 15 .

9.4. ANN versus Integrated ANN-GA. The minimum predicted Dilution value of the ANN model is 9.353; it can be concluded that both integration systems have given the more optimum result of the dilution compared to ANN model. Consequently, integrated ANNSA type A and integrated ANN-GA type A are well within the limits of standard dilution.

9.5. GA versus Integrated $A N N-G A$. The minimum predicted dilution value of the GA was 10.375 . Therefore, with dilution 9.745, it can be concluded that both integrated systems have given the more minimum of the Dilution compared to GA technique.

9.6. Second Objective: Evaluation of the Optimal Process Parameters. For the second objective of this study, the optimal values of the integrated ANN-GA type A and integrated ANN-SA type B for each process parameter are within the range of minimum and maximum values of experimental design; thus, this study concludes that the second objective of this study is fulfilled.

9.7. Third Objective: Evaluation of the Number of Iteration. Integrated ANN-GA type A and integrated ANN-GA type $B$ are approximately the same as or lower than the number of iterations by GA; thus, this study concludes that the third objective of this study is fulfilled.

\section{Conclusion}

(1) A five-level-five-factor full factorial design matrix based on central composite routable design technique was used for building the mathematical model.

(2) ANN tool box available in MATLAB was used for the prediction of bead geometry.

(3) In this study two models, ANN and GA are used for prediction and optimization of bead geometry.

(4) In GMAW process bead geometry plays an important role in economising the material. This study effectively used the integration method for optimization.
(5) This study proposed two integration systems, integrated ANNGA type A and integrated ANN-GA type $\mathrm{B}$, in order to estimate the optimal solutions of process parameters that lead to minimum dilution found to satisfy three conditions. Overall, integrated ANN-GA type A and integrated ANN-GA type B have been very effective in estimating optimum values of dilution compared to experimental data and regression method. In the second process the optimal value of process parameters recommended by integrated type $A$ and integrated type $B$ are well within the range of minimum and maximum value of process parameters of experimental design. In the third issue it was found that the proposed integration system satisfies the number of iterations than the single system of optimization.

\section{References}

[1] P. K. Palani and N. Murugan, "Prediction of delta ferrite content and effect of welding process parameters in claddings by FCAW," Materials and Manufacturing Processes, vol. 21, no. 5, pp. 431-438, 2006.

[2] T. Kannan and N. Murugan, "Prediction of Ferrite Number of duplex stainless steel clad metals using RSM," Welding Journal, vol. 85, no. 5, pp. 91-s-99-s, 2006.

[3] N. Murugan and V. Gunaraj, "Prediction and control of weld bead geometry and shape relationships in submerged arc welding of pipes," Journal of Materials Processing Technology, vol. 168, no. 3, pp. 478-487, 2005.

[4] I. S. Kim, K. J. Son, Y. S. Yang, and P. K. D. V. Yaragada, "Sensitivity analysis for process parameters in GMA welding processes using a factorial design method," International Journal of Machine Tools and Manufacture, vol. 43, no. 8, pp. 763769, 2003.

[5] W. G. Cochran and G. M. Coxz, Experimental Design, John Wiley \& Sons, New York, NY, USA, 1987.

[6] S. Karaoğlu and A. Seçgin, "Sensitivity analysis of submerged arc welding process parameters," Journal of Materials Processing Technology, vol. 202, no. 1-3, pp. 500-507, 2008.

[7] P. K. Ghosh, P. C. Gupta, and V. K. Goyal, "Stainless steel cladding of structural steel plate using the pulsed current GMAW process," Welding Journal, vol. 77, no. 7, pp. 307-s-314-s, 1998.

[8] V. Gunaraj and N. Murugan, "Prediction and comparison of the area of the heat-affected zone for the bead-on-plate and beadon-joint in submerged arc welding of pipes," Journal of Materials Processing Technology, vol. 95, no. 1-3, pp. 246-261, 1999.

[9] D. C. Montgomery, Design and Analysis of Experiments, John Wiley \& Sons, 2003.

[10] T. Kannan and J. Yoganandh, "Effect of process parameters on clad bead geometry and its shape relationships of stainless steel claddings deposited by GMAW,' International Journal of Advanced Manufacturing Technology, vol. 47, pp. 1083-1095, 2010.

[11] P. Dutta and D. K. Pratihar, "Modeling of TIG welding process using conventional regression analysis and neural networkbased approaches," Journal of Materials Processing Technology, vol. 184, no. 1-3, pp. 56-68, 2007. 
[12] H. Ates, "Prediction of gas metal arc welding parameters based on artificial neural networks," Materials and Design, vol. 28, no. 7, pp. 2015-2023, 2007.

[13] J. I. Lee and K. W. Um, "Prediction of welding process parameters by prediction of back-bead geometry," Journal of Materials Processing Technology, vol. 108, no. 1, pp. 106-113, 2000.

[14] A. Mukhopadhyay and A. Iqbal, "Prediction of mechanical properties of hot rolled, low-carbon steel strips using artificial neural network," Materials and Manufacturing Processes, vol. 20, no. 5, pp. 793-812, 2005.

[15] D. K. Panda and R. K. Bhoi, "Artificial neural network prediction of material removal rate in electro discharge machining," Materials and Manufacturing Processes, vol. 20, no. 4, pp. 645672, 2005.

[16] D. S. Nagesh and G. L. Datta, "Prediction of weld bead geometry and penetration in shielded metal-arc welding using artificial neural networks," Journal of Materials Processing Technology, vol. 123, no. 2, pp. 303-312, 2002.

[17] K. Manikya Kanti and P. Srinivasa Rao, "Prediction of bead geometry in pulsed GMA welding using back propagation neural network," Journal of Materials Processing Technology, vol. 200, no. 1-3, pp. 300-305, 2008.

[18] A. M. Zain, H. Haron, and S. Sharif, “Optimization of process parameters in the abrasive waterjet machining using integrated SA-GA," Applied Soft Computing Journal, vol. 11, no. 8, pp. 53505359, 2011.

[19] F. Cus and J. Balic, "Optimization of cutting process by GA approach," Robotics and Computer-Integrated Manufacturing, vol. 19, no. 1-2, pp. 113-121, 2003.

[20] A. M. Zain, H. Haron, and S. Sharif, "Estimation of the minimum machining performance in the abrasive waterjet machining using integrated ANN-SA," Expert Systems with Applications, vol. 38, no. 7, pp. 8316-8326, 2011.

[21] P. Sahoo, "Optimization of turning parameters for surface roughness using RSM and GA," Advances in Production Engineering and Management, vol. 6, no. 3, pp. 197-208, 2011.

[22] F. Kolahan and M. Heidari, "A new approach for predicting and optimizing weld bead geometry in GMAW," World Academy of Science, Engineering and Technology, vol. 59, pp. 138-141, 2009.

[23] K. Siva, N. Murugan, and R. Logesh, "Optimization of weld bead geometry in plasma transferred arc hardfaced austenitic stainless steel plates using genetic algorithm," International Journal of Advanced Manufacturing Technology, vol. 41, no. 1-2, pp. 24-30, 2009. 

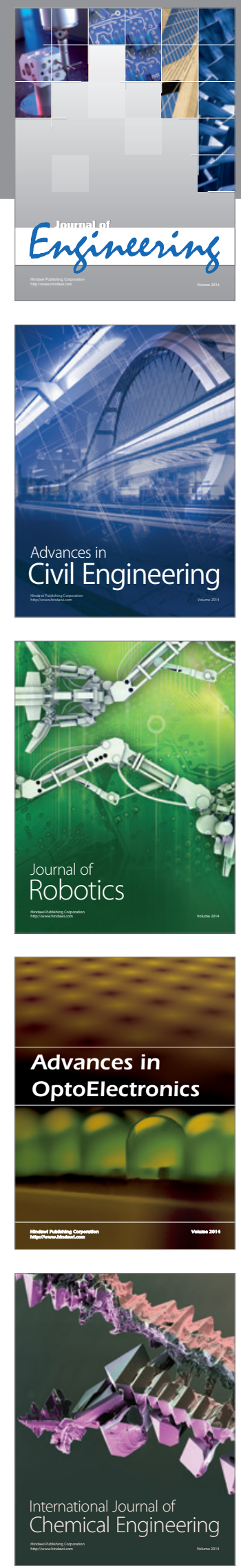

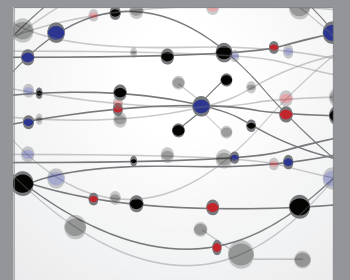

The Scientific World Journal
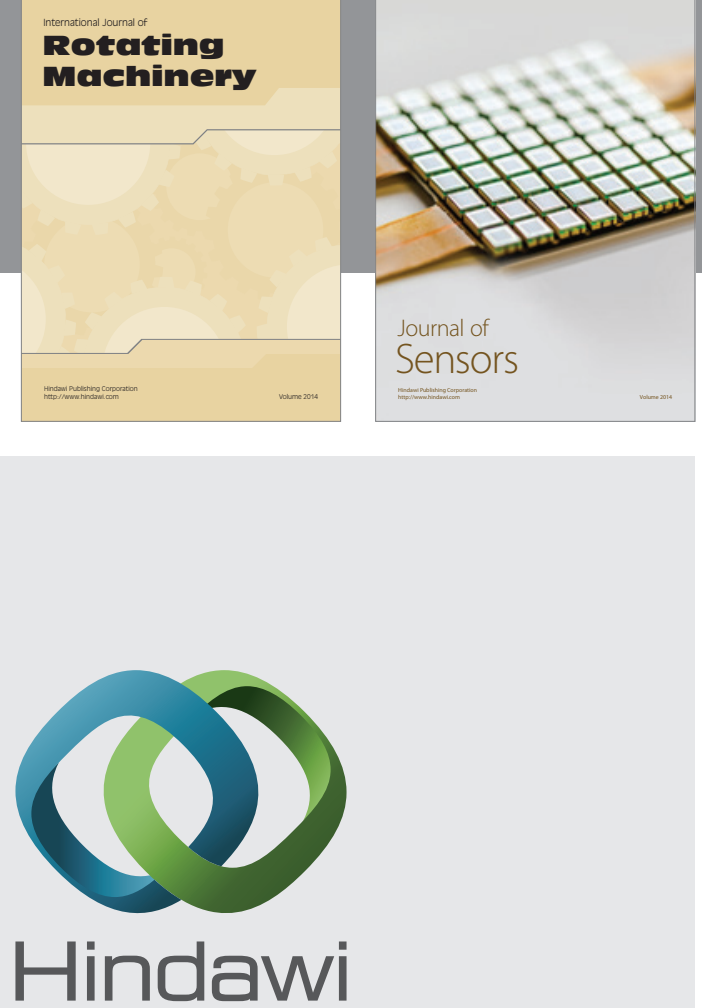

Submit your manuscripts at http://www.hindawi.com
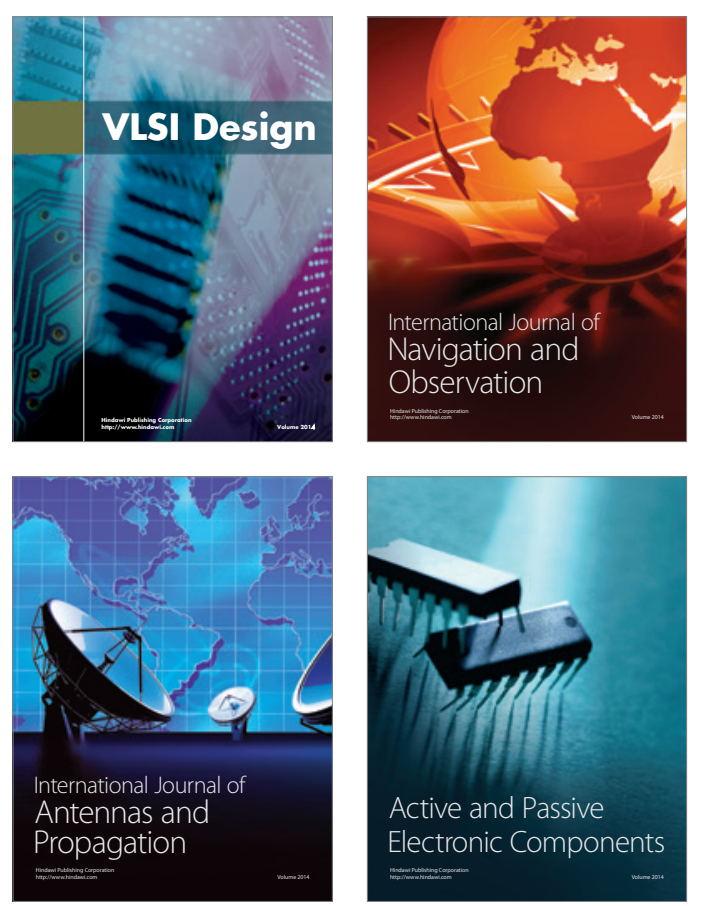
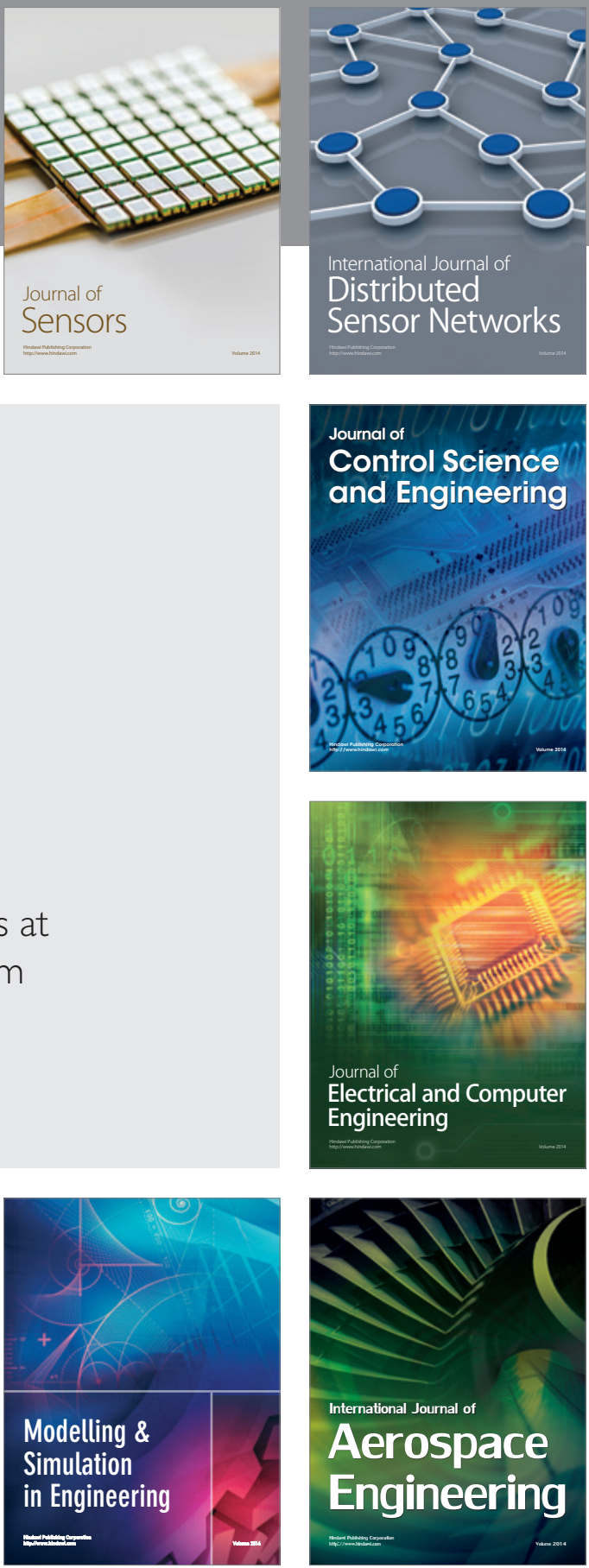

Journal of

Control Science

and Engineering
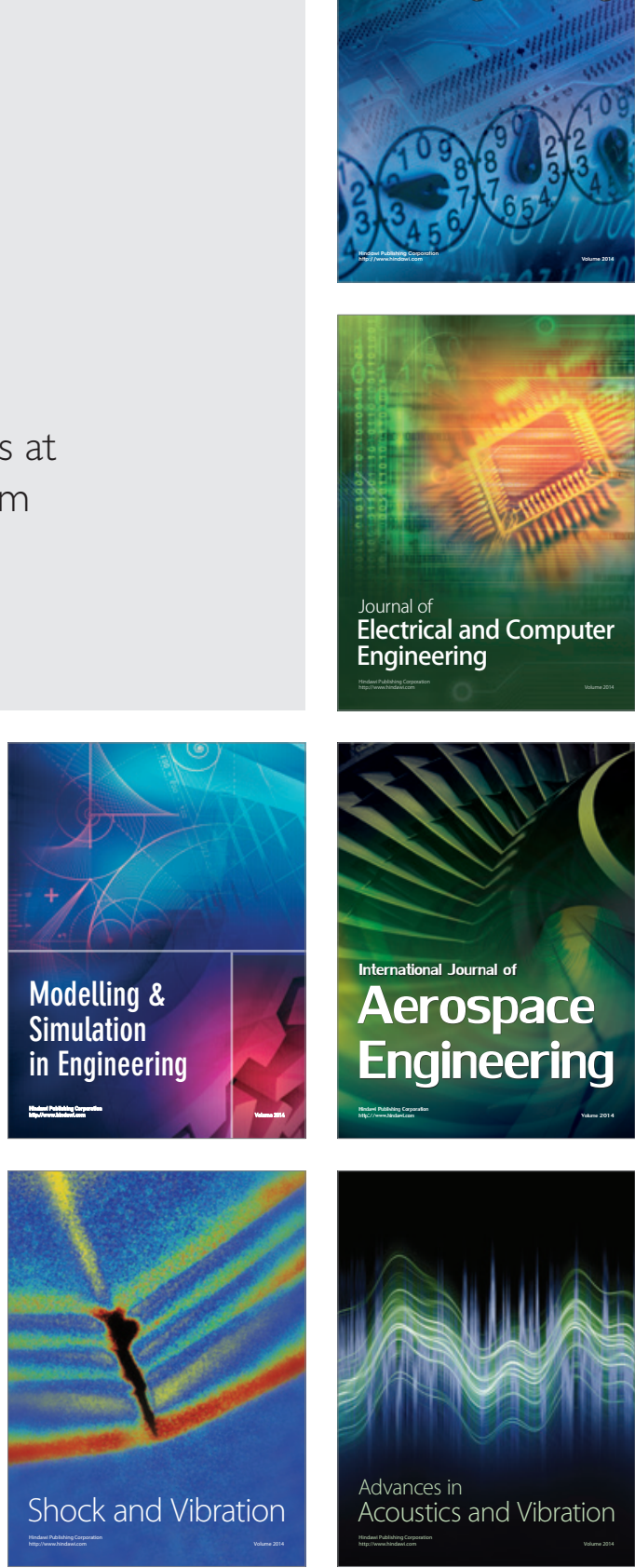